Research Article

\title{
Vibration Characteristics of Rolling Mill System under Constraints of the Nonlinear Spring Force and Friction Force from Hydraulic Cylinder
}

\author{
Zhaolun Liu $\mathbb{D}^{1},{ }^{1}$ Guixiang Pan, ${ }^{2}$ Jiahao Jiang, ${ }^{1}$ and Bin Liu $^{2}$ \\ ${ }^{1}$ School of Information Science and Engineering, Yanshan University, Qinhuangdao 066004, China \\ ${ }^{2}$ School of Electrical Engineering, Yanshan University, Qinhuangdao 066004, China \\ Correspondence should be addressed to Zhaolun Liu; liuzhaolun@ysu.edu.cn
}

Received 23 October 2020; Revised 9 December 2020; Accepted 9 January 2021; Published 19 January 2021

Academic Editor: Luca Pugi

Copyright (c) 2021 Zhaolun Liu et al. This is an open access article distributed under the Creative Commons Attribution License, which permits unrestricted use, distribution, and reproduction in any medium, provided the original work is properly cited.

Considering the two kinds of nonlinear constraints of rolling mill hydraulic cylinder, spring force and friction force, the vibration model of rolling mill system is established. The amplitude frequency response equations are obtained by using the average method. Comparing the time history curves of vertical vibration displacement of rolling mill system under the nonlinear spring force and friction force, the amplitude frequency characteristic curves are simulated. The external excitation amplitude is viewed as the bifurcation parameter, and the system bifurcation response changing with the external excitation amplitude is analyzed. The influence of the external excitation amplitude on the system stability is studied. The results indicate that the increase of the nonlinear spring force makes the rolling mill system's unstable area to become wider, and the influence on the rolling mill system of nonlinear friction force behaves as the damping characteristics; the vibration of rolling mill system is alternating between the periodic, period-doubling, and the chaotic motion. The research results provide a theoretical support for restraining the vibration of the rolling mill system in the actual production process.

\section{Introduction}

With the rapid development of science and technology, strip rolling mill in the field of heavy industrial machinery plays an increasingly important role [1]. However, the vibration of rolling mill not only limits the rolling speed, but impacts the accuracy. It affects the quality of rolling products and hazards the safety of mill production [2-4].

The research on vibration of plate and strip rolling mill has lasted for nearly half a century, and scholars have studied it from different angles [5-7]. Yarita et al. constructed a simple mass-spring-damper vibration system and provided a method to estimate the spring constants and damping coefficients of the system [8]. Sun et al. studied the influence of the tension factor on the nonlinear vibration of the rolling mill [9]. The stability of the rolling mill vibration system was analyzed by changing the external excitation frequency. They found that the rolling speed and the thickness of the strip have great influence on the stability of the system. Yun et al. developed a suitable model to study the vibration of mill system. This model reflected the dynamic relationship among the rolling parameters. They announced that the selfexcited vibration occurring in rolling process is the consequence of the interaction between the structural dynamics of mill stand and the dynamics of the rolling process itself [10]. Yang et al. took into account the interaction between the roller and the rolled piece and established a vertical vibration model of roll system based on the dynamic friction equation of roll gap [11]. The vertical vibration mechanism of cold rolling mill is studied under the influence of the dynamic friction mechanism between the workpiece and roll. Fan et al. introduced the Duffing oscillator and the parameters of the excitation system and established the roll system vertical vibration model [12]. The bifurcation characteristics of the rolling mill system amplitude in the nonlinear parameter variation are analyzed by the 
simulation. Bayón et al. considered the nonlinear friction coefficient and nonlinear equivalent stiffness of hydraulic cylinder are caused by the change of velocity and dynamic load [13]. The dynamic characteristics of the hydraulic system are also analyzed by controlling the electric motor speed [14]. The results show that the rotation angle of the base plate gradually becomes more negative during the open-loop control because of the difference in the area of the hydraulic cylinder. The above researches were from the background of rolling mill structure and rolling process. The rolling mill hydraulic cylinder is a typical nonlinear component in the rolling mill system, and the influence of nonlinear constraints on the vibration behavior of the rolling mill system cannot be ignored. The coefficient of friction between the cylinder and the piston will change due to the periodic change of the vibration velocity of the hydraulic cylinder, and the friction exhibits nonlinear. In the running process of the hydraulic cylinder system, under the action of the oil source pressure and the load pressure, the dynamic hydraulic spring can be formed due to the compressibility of the oil [15]. The nonlinearity of the spring force makes the natural frequency of the system unstable, and the stability of the response area becomes complicated [16]. The spring force will cause the nonlinear vibration of the hydraulic system under certain conditions. Zhang et al. analyzed the transverse nonlinear vibrations of a rotating flexible disk subjected to a rotating point force with a periodically varying rotating speed. The dynamics of the cantilevered shellshaped workpiece was studied under time-delay effects, parametric excitation, and forcing excitation $[17,18]$. Zhou et al. investigated the nonlinear dynamics of a thin-plate workpiece during milling process with cutting force nonlinearities [19]. Numerical methods are used to find the periodic and chaotic oscillations of the cantilevered thinplate workpiece. Andrea et al. proposed an innovative controlled hydraulic pump solution to drastically improve the efficiency and to reduce the overall encumbrances, maintaining very high performance in terms of dynamical behavior and corresponding frequency response [20]. Valigi et al. presented a numerical model combining Orowan theory to predict the working parameters of full film lubrication cold rolling process. Using it, we can forecast the thickness reduction of the metal sheet and the pressure trend, and the rolling mill process parameters can be regulated to obtain a specific output thickness [21]. It is significant to establish a more practical vibration model of rolling mill system for the analysis of roll system vibration.

In this paper, the rolling mill system with nonlinear spring force and friction force constraints is studied. Considering the nonlinear factors such as spring force and friction force of hydraulic cylinder, the vibration model of rolling mill system is established based on the two kinds of nonlinear constraint. The influence of nonlinear spring force, friction force, and the initial displacement of piston rod on the vibration behavior of rolling mill system and the bifurcation characteristics under the action of external excitation amplitude are studied.

\section{Vibration Model of Rolling Mill System}

2.1. Vibration Model of Rolling Mill System under Nonlinear Constraints. Based on the production line of Hebei Iron and Steel Group Chengde branch, the data of all the simulation experiments are derived from the structural parameters of the 1780 rolling mill and the parameters of the rolling process. Figure 1 is the four-roller mill physical map, in which 1 is the hydraulic cylinder, 2 is the frame, 3 is the upper supporting roll, 4 is the upper work roll, 5 is the lower work roll, and 6 is the lower supporting roll.

According to the viewpoint of nonlinear dynamics, the effect of nonlinear spring force and friction force those from hydraulic cylinder on the vibration of rolling mill system is studied [22]. In order to simplify the research, taking into account the symmetry of the structure of the four-roller mill, only the upper roll of rolling mill system is analyzed. The vibration model of rolling mill system with nonlinear spring force and friction force constraints is established, and the diagram is shown in Figure 2.

Based on the generalized Lagrange principle, the dynamic equation of rolling mill system can be expressed as [23]

$m \ddot{x}+c \dot{x}+k x+\alpha\left(k_{1} x+k_{3} x^{3}\right)+\beta\left(c_{0}-c_{1} \dot{x}+c_{3} \dot{x}^{3}\right)=F \cos (\omega t)$,

where $m$ indicates the equivalent mass of the upper supporting roller, the upper working roller, and the bearing seat of the four-roller mill. $c$ is the equivalent damping system of the rolling mill. $k$ is the equivalent stiffness of the rolling mill system. $x$ is the displacement of the piston rod. $F$ is equivalent to the load force. In order to study the influence of different nonlinear constraints on the roll system vibration behavior, the nonlinear spring force and friction force constraints of the equation are known as the coefficients $\alpha$ and $\beta$.

2.2. Nonlinear Spring Force. The hydraulic spring stiffness is the nonlinear spring stiffness of the liquid, which is formed when the volume of the fluid is compressed by the external force, when the hydraulic cylinder working chamber is completely closed. In this paper, the double-acting singlepiston hydraulic cylinder used in rolling mill industry is analyzed. The spring stiffness of the rolling mill hydraulic cylinder is composed of the hydraulic oil stiffness and the stiffness of the piston rod. The bulk modulus of the piston rod is nearly 100 times that of the bulk modulus of hydraulic oil, so it is regarded as a rigid body. So the spring stiffness of the hydraulic cylinder system of the rolling mill is produced by the spring stiffness of the hydraulic oil [24]. The oil in the two cavities is always under pressure and in compression state, so the total hydraulic spring stiffness of the hydraulic cylinder can be equivalent to the parallel of the hydraulic spring stiffness of the two cavities [25]. 


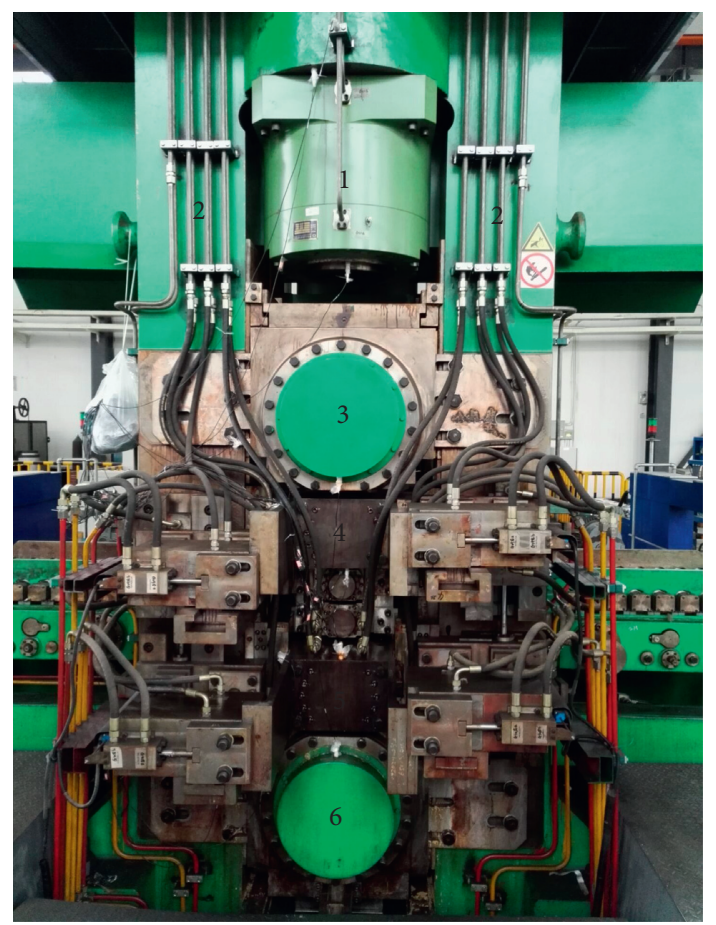

FIgURE 1: The four-roller mill physical map.

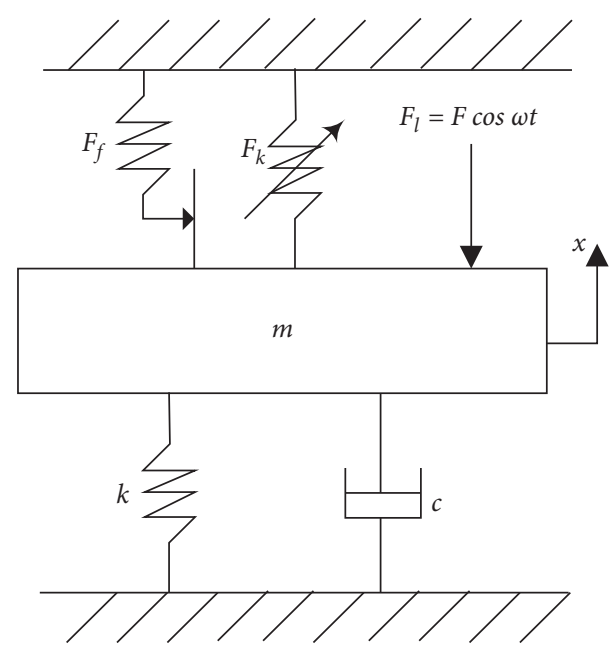

FIgURE 2: The vibration model of the rolling mill system.

$k(x)=\frac{\beta_{e} A_{1}^{2}}{V_{1}}+\frac{\beta_{e} A_{2}^{2}}{V_{1}}=\frac{\beta_{e} A_{1}^{2}}{A_{1}(L 1+x)+V_{l 1}}+\frac{\beta_{e} A_{2}^{2}}{A_{2}(L-L 1-x)+V_{l 2}}$,

where $\beta_{e}$ is the volume elastic modulus of hydraulic cylinder oil, $A_{1}\left(A_{2}\right)$ is the hydraulic cylinder without (with) the effective area of the piston side of the rod cavity, $L$ is the total stroke of the hydraulic cylinder, $L 1$ is the initial position of the piston rod, $V_{l 1}\left(V_{l 2}\right)$ is the oil volume in the tubing between the inlet valve and the rodless (rod) chamber, and $V_{l 1}\left(V_{l 2}\right)$ has the small relative to the two oil chamber.
The vibration displacement of the spring stiffness formula at the working point is $x$. At the origin, when $x=0$, the Taylor series can be expressed as

$$
k(x)=k(0)+\dot{k}(0) x+\frac{\ddot{k}(0)}{2 !} x^{2}+o\left(x^{2}\right) .
$$

Make $k(0)=k_{1}, \dot{k}(0)=k_{2}$, and $(\ddot{k}(0) t / 2 !)=k_{3}$; then

$$
k(x)=k_{1}+k_{2} x+k_{3} x^{2}+o\left(x^{2}\right) .
$$

Nonlinear spring force can be expressed as

$$
k(x)=k(0)+\dot{k}(0) x+\frac{\ddot{k}(0)}{2 !} x^{2}+o\left(x^{2}\right) .
$$

Because the elastic potential energy of spring is symmetrical, $U$ can be expressed as

$$
U=\frac{1}{2} k_{1} x^{2}+\frac{1}{4} k_{3} x^{4}
$$

The type (6) derivation, the spring force of the hydraulic cylinder, can be expressed as

$$
F_{s}(x)=\frac{\mathrm{d} U}{\mathrm{~d} x}=k_{1} x+k_{3} x^{3} .
$$

2.3. Nonlinear Friction Force. In the hydraulic cylinder, friction occurs between the piston rod and the cylinder wall in order to achieve the transfer of energy. When it works, it shows the strong nonlinear effect, which can cause the rolling mill system to produce the flutter phenomenon, which makes the rolling mill system to produce the nonlinear vibration [24]. Therefore, the influence of the friction force on the dynamic characteristics of rolling mill system cannot be ignored. The friction coefficient between the rolling mill hydraulic cylinder piston rod and the cylinder wall is an expression related to speed [26]. Therefore, the friction coefficient can be expressed as

$$
\mu=\mu_{s} \operatorname{sgn}(\dot{x})-\lambda_{1} \dot{x}+\lambda_{2} \dot{x}^{3},
$$

where $\lambda_{1}=\left(3\left(\mu_{s}-\mu_{m}\right) / 2 v_{m}\right)$ and $\lambda_{2}=\left(\mu_{s}-\mu_{m}\right) / 2 v_{m}^{3}$, where $\mu_{s}$ is the static friction coefficient, $\mu_{m}$ is the maximum dynamic friction factor, and $\nu_{m}$ is the vibration velocity when the friction coefficient is $\mu_{m}$.

The friction force of the hydraulic cylinder of the rolling mill can be expressed as

$$
F_{f}(\dot{x})=p \times \mu=p\left(\mu_{s} \operatorname{sgn}(\dot{x})-\lambda_{1} \dot{x}+\lambda_{2} \dot{x}^{3}\right)=c_{0}-c_{1} \dot{x}+c_{3} \dot{x}^{3},
$$

where $p$ is the pressure between the piston rod and the cylinder wall, and $p$ depends on the material of the rolling mill hydraulic cylinder, the hardness of the sealing material, and the radial component of the load where $c_{0}=p \times \mu_{s} \operatorname{sgn}(\dot{x}), c_{1}=p \times \lambda_{1}$, and $c_{3}=p \times \lambda_{2} . F_{s}(x)$ and $F_{f}(\dot{x})$ are the foundation of the vibration model of the rolling mill system. 


\section{Rolling Mill System Response}

In order to facilitate the calculation, the kinetic equation (11) can be simplified as

$$
\begin{aligned}
\ddot{x} & +2 \xi \omega_{0} \dot{x}+\omega_{0}^{2} x+\alpha\left(\rho_{1} x+\gamma_{1} x^{3}+\rho_{2} x+\gamma_{2} x^{3}\right) \\
& +\beta\left(c_{1}^{*} \dot{x}+c_{3}^{*} \dot{x}^{3}\right)=F^{*} \cos (\omega t),
\end{aligned}
$$

where $\xi=\left(c / 2 m \omega_{0}\right), \quad \omega_{0}=\sqrt{k / m}, \quad \rho_{1}=\left(\beta_{e} A_{1} / m L_{1}\right)$, $\rho_{2}=\left(\beta_{e} A_{2} / m\left(L-L_{1}\right)\right), \quad \gamma_{1}=\left(\beta_{e} A_{1} / m L_{1}^{3}\right)$, $\gamma_{2}=\left(\beta_{e} A_{2} / m\left(L-L_{1}\right)^{3}\right), \quad c_{1}^{*}=-\left(c_{1} / m\right), \quad c_{3}^{*}=\left(c_{3} / m\right)$, $F^{*}=(F / m)$, and $\left(c_{0} / m\right) \longrightarrow 0$.

Taking into account the primary resonance with the external excitation, make $\omega^{2}=\omega_{0}^{2}(1+\varepsilon \sigma)$, and then

$$
\ddot{x}+\omega^{2} x=\varepsilon f(x, \dot{x}),
$$

where $\varepsilon$ is the nonlinear term coefficient and $\sigma$ is the frequency tuning factor:

$$
\begin{aligned}
f(x, \dot{x})= & F^{*} \cos (\omega t)+\varepsilon \sigma \omega_{0}^{2} x-2 \xi \omega_{0} \dot{x} \\
& -\alpha\left(\rho_{1} x+\gamma_{1} x^{3}+\rho_{2} x+\gamma_{2} x^{3}\right)-\beta\left(c_{1}^{*} \dot{x}+c_{3}^{*} \dot{x}^{3}\right) .
\end{aligned}
$$

When $\varepsilon=0$, the derived system of equation (11) is a linear conservative system, and the solution of the derived system and its derivative can be expressed as

$$
\left\{\begin{array}{l}
x=a \cos \left(\omega_{0} t-\theta\right), \\
\dot{x}=-a \omega_{0} \sin \left(\omega_{0} t-\theta\right) .
\end{array}\right.
$$

By substituting equation (13) into equation (11), the following expression can be obtained:

$$
\left\{\begin{array}{l}
\dot{a}=-\frac{\varepsilon}{\omega_{0}} f(x, \dot{x}) \sin \phi, \\
\dot{\theta}=\frac{\varepsilon}{\omega_{0} a} f(x, \dot{x}) \cos \phi,
\end{array}\right.
$$

where $\phi=\omega t-\theta$. When the parameter $\varepsilon$ is sufficiently small, $a$ and $\theta$ are slowly varying functions near the constant. The right term of equation (11) is approximately replaced by the average value of one cycle of $\phi$, and it is also believed that $a$ and $\theta$ remain unchanged in one cycle of $\phi$. Thus the equation obtained is called the mean equation of the original equation:

$$
\left\{\begin{array}{l}
\dot{a}=-\frac{\varepsilon}{2 \omega_{0}} Q(a, \theta), \\
\dot{\theta}=\frac{\varepsilon}{2 \omega_{0} a} P(a, \theta),
\end{array}\right.
$$

where $Q(a, \theta)$ and $P(a, \theta)$ can be expressed as

$$
\left\{\begin{array}{l}
Q(a, \theta)=\frac{1}{\pi} \int_{0}^{2 \pi} f(x, \dot{x}) \sin \phi d \phi, \\
P(a, \theta)=\frac{1}{\pi} \int_{0}^{2 \pi} f(x, \dot{x}) \cos \phi d \phi .
\end{array}\right.
$$

By substituting equation (12) into equation (16), the following expression can be obtained:

$$
\left\{\begin{array}{l}
Q(a, \theta)=F^{*} \sin \theta+2 \xi \omega_{0}^{2} a+\beta\left(c_{1}^{*} a \omega_{0}+0.75 c_{3}^{*} a^{3} \omega_{0}^{3}\right) \\
P(a, \theta)=F^{*} \cos \theta+\varepsilon \sigma \omega_{0}^{2} a-\alpha\left(\begin{array}{c}
\rho_{1} a+0.75 \gamma_{1} a^{3} \\
+\rho_{2} a+0.75 \gamma_{2} a^{3}
\end{array}\right)
\end{array}\right.
$$

By substituting equation (17) into equation (15), the following expression can be obtained:

$$
\left\{\begin{array}{c}
\dot{a}=-\frac{\varepsilon}{2 \omega_{0}}\left(-F^{*} \sin \theta+2 \xi \omega_{0}^{2} a+\beta c_{1}^{*} a \omega_{0}+0.75 \beta c_{3}^{*} a^{3} \omega_{0}^{3}\right), \\
\dot{\theta}=\frac{\varepsilon}{2 \omega_{0} a}\left(\begin{array}{c}
F^{*} \cos \theta+\varepsilon \sigma \omega_{0}^{2} a-\alpha \rho_{1} a-0.75 \alpha \gamma_{1} a^{3} \\
-\alpha \rho_{2} a-0.75 \alpha \gamma_{2} a^{3}
\end{array}\right) .
\end{array}\right.
$$

When $\dot{a}=0, \dot{\theta}=0$, after eliminating $\theta$ in equation (18), the amplitude frequency equation of the system can be obtained:

$$
\left(\begin{array}{c}
2 \xi \omega_{0}^{2} a+\beta c_{1}^{*} a \omega_{0} \\
+0.75 \beta c_{3}^{*} a^{3} \omega_{0}^{3}
\end{array}\right)^{2}+\left(\begin{array}{c}
\varepsilon \sigma \omega_{0}^{2} a-\alpha \rho_{1} a-0.75 \alpha \gamma_{1} a^{3} \\
-\alpha \rho_{2} a-0.75 \alpha \gamma_{2} a^{3}
\end{array}\right)^{2}=\left(F^{*}\right)^{2} .
$$

Equation (19) is the amplitude frequency characteristic equation of rolling mill system, which is the basis of research on the vibration behavior of rolling mill system with nonlinear spring force and friction force constraints. The average method is used to solve the rolling mill roll system vibration system under the nonlinear constraint of the hydraulic cylinder, and the dynamic amplitude frequency response equation of the vibration system is obtained.

\section{Vibration Characteristics of Rolling Mill System}

Taking the parameters of the 1780 strip rolling mill in a certain factory as an example, the vertical vibration model of the roller system under the nonlinear action of the hydraulic cylinder is solved by using the actual parameters of the rolling mill. Based on the actual numerical experiments, the effects of the nonlinear spring force and the nonlinear 
friction force on the dynamic characteristics of the rolling mill system are studied. The parameters in the system model are shown in Table 1.

4.1. Time-Domain Characteristics. The vibration of rolling mill system is influenced by the nonlinear spring force and nonlinear friction force of the hydraulic cylinder of rolling mill. By taking different nonlinear spring force and friction coefficient, the variation law of time-domain response is simulated and analyzed. The simulation results are shown in Figures 3-5.

As is shown in Figures 3(a) and 3(b), with the increase of nonlinear friction, the peaks and troughs of the vertical vibration curve of the rolling mill system showed irregular vibration. It can be seen from Figures 3(b) and 3(c) that when the nonlinear spring force is increased, the vibration curve of rolling mill system has various irregular vibration. With the increase of the nonlinear binding force, the more irregular vibration of the milling mill system occurred, and this affects the stability of the system. From Figure 3(a) to Figure 3(c), when the nonlinear friction force is increased, the amplitude of the vibration displacement has no obvious change, but the amplitude of the vibration displacement is greatly reduced when the nonlinear spring force is increased. The above conclusions provide data support for the manufacture of rolling mill hydraulic cylinder. It is helpful to improve the stability of the rolling mill by selecting the appropriate nonlinear spring stiffness coefficient and friction coefficient.

4.2. Frequency-Domain Characteristics. The influence of different rolling parameters on the vibration of rolling mill system is studied. By using the actual parameters listed in Table 1, the amplitude frequency characteristics of the system are simulated, and then the influence of the initial displacement of the piston rod, the nonlinear friction force, and the nonlinear spring force on the rolling mill system vibration are analyzed.

Figure 4 shows the different amplitude frequency characteristic curves of hydraulic cylinder piston rod under different initial displacement. The amplitude frequency characteristic curves are very sensitive to the initial displacement of the piston rod. The initial displacement is slightly smaller, the amplitude-frequency curve moves to the right, the rolling natural frequency is slightly larger, and the curvature of the amplitude-frequency characteristic curve becomes larger, which means the instability region of the system becomes wider. The stability of the system can be enhanced by properly adjusting the initial displacement of the hydraulic cylinder of the rolling mill, which provides technical support for the manufacture of hydraulic cylinder.

The comparison of different nonlinear friction coefficients in Figure 5 shows that the nonlinear friction in the system is equivalent to the damping of the system. With the increase of $\beta$, amplitude frequency characteristic curve of the amplitude decreases. In a certain range, with the increase of nonlinear friction, the vibration of the rolling mill system increases.
TABLE 1: Vibration parameters of rolling mill system.

\begin{tabular}{lc}
\hline Parameters & Values \\
\hline Mass of up rolls $(m)$ & $1.44 \times 10^{5} \mathrm{~kg}$ \\
Stiffness of up rolls $(k)$ & $2.35 \times 10^{10} \mathrm{~N} / \mathrm{m}$ \\
Damping of up rolls $(c)$ & $2.54 \times 10^{6} \mathrm{~N} \cdot \mathrm{s} / \mathrm{m}$ \\
Total stroke of hydraulic cylinder $(L)$ & $0.11 \mathrm{~m}$ \\
Piston side area $\left(A_{1}\right)$ & $0.6361 \mathrm{~m}^{2}$ \\
Piston side area $\left(A_{2}\right)$ & $0.3243 \mathrm{~m}^{2}$ \\
Pressure $(p)$ & $0.04 \mathrm{MN}$ \\
Oil elastic modulus $\left(\beta_{e}\right)$ & $1.6 \times 10^{9} \mathrm{~Pa}$ \\
Amplitude of external excitation $\left(F_{0}\right)$ & $0.6 \mathrm{MN}$ \\
Nonlinear term coefficient $(\varepsilon)$ & 0.01 \\
Maximum dynamic friction factor $\left(\mu_{m}\right)$ & 0.01 \\
Static friction coefficient $\left(\mu_{s}\right)$ & 0.02 \\
Vibration velocity $\left(v_{m}\right)$ & $0.01 \mathrm{~m} / \mathrm{s}$ \\
\hline
\end{tabular}

The comparison of different nonlinear spring coefficients in Figure 6 shows that with the increase of the nonlinear spring force coefficient, the amplitude frequency curves of the system moves to the right, and the inherent frequency of the rolling mill becomes larger. At the same time, the bending degree of the amplitude frequency characteristic curve becomes larger, the unstable region of the system becomes wider, and the rolling process of rolling mill system will be easier to lose stability.

4.3. Bifurcation Characteristics. The amplitude of excitation amplitude is used as the bifurcation parameter, the change law of the system bifurcation response is analyzed, and the influence of the amplitude of the external excitation on the stability of the system is studied.

It can be seen from Figure 7 that when the external excitation takes different values, the system has different degrees of bifurcation phenomena. The bifurcation diagram shows the single-value curve, the multivalued curve, and the infinite valued curve. The solutions of the system equations may be single solution, multiple solutions, and infinite solutions. That is, the vibration of rolling mill system is changed between one period, double period, and the chaotic motion. When the external excitation is $F=5.91 \times 10^{5} \mathrm{~N}$, the simulation results are shown in Figure 8: the phase trajectory is repeated in a finite region, which is a closed curve. There is only one isolated point in a certain area of the Poincare section, which indicates that the vibration of rolling mill system is periodic motion. When the external excitation is $F=6.012 \times 10^{5} \mathrm{~N}$, the simulation results are shown in Figure 9: the phase trajectory is still repeated in a limited area, which is a closed curve. There are two isolated points in a certain area of the Poincaré section, which indicates that the vibration of rolling mill system is 2 times of the periodic motion. When the external excitation is $F=6.15 \times 10^{5} \mathrm{~N}$, the simulation results are shown in Figure 10: the phase trajectory is still repeated in a limited area, which is a closed curve. There are three isolated points in a certain area of the Poincaré section, which indicates that the vibration of rolling mill system is 3 times of the periodic motion. When the external excitation is $F=6.27 \times 10^{5} \mathrm{~N}$, the simulation results are shown in Figure 11: the phase 


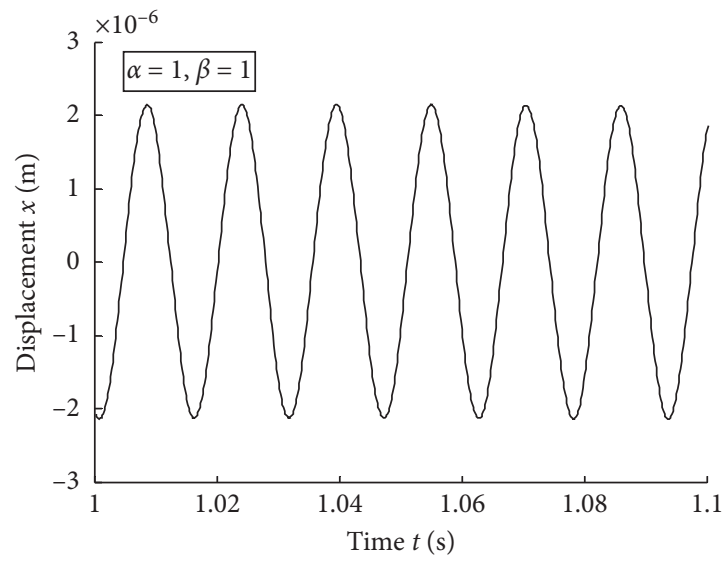

(a)

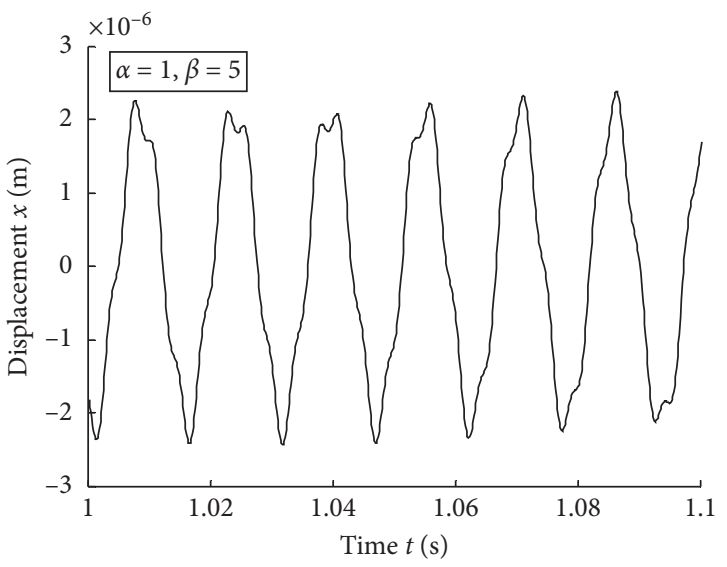

(b)

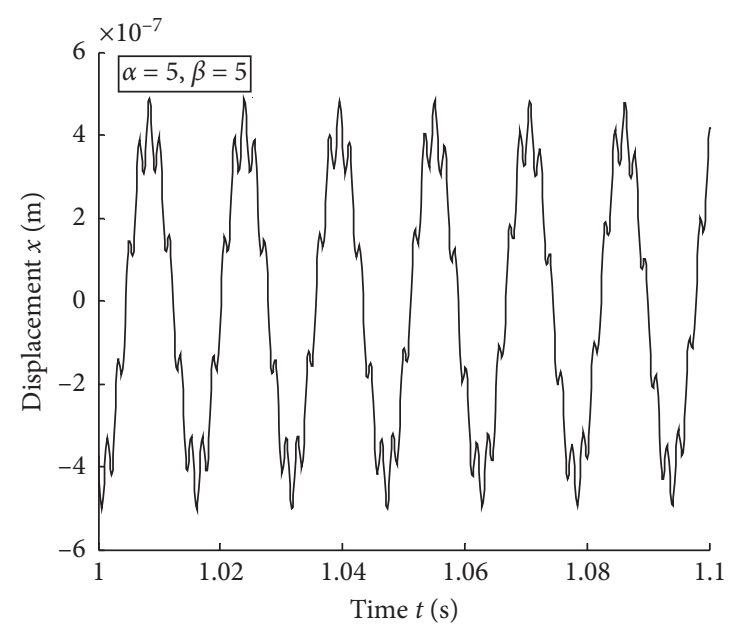

(c)

Figure 3: The vertical vibration curve of rolling mill system.

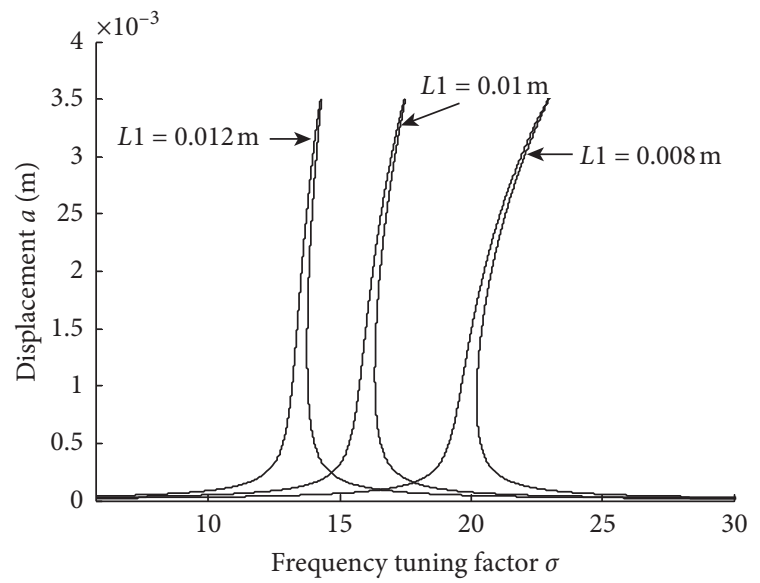

FIgURE 4: Amplitude frequency characteristics of different initial displacement.

trajectory is no longer a closed curve, and the corresponding Poincaré section is a discrete point in a certain region. At this time, the vibration of rolling mill system is chaotic motion

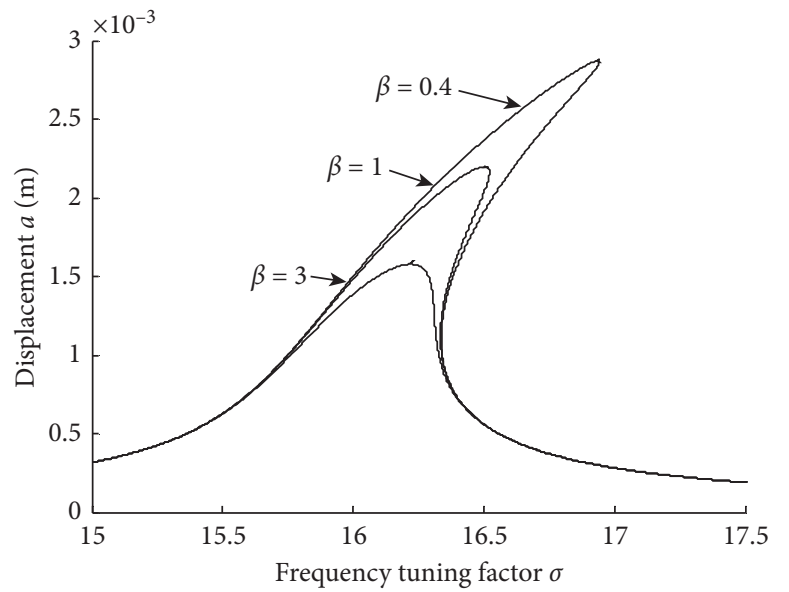

Figure 5: The influence of nonlinear friction coefficient on amplitude frequency characteristic curve.

state. In this paper, the data of all the simulation experiments are derived from the structural parameters of the 1780 rolling mill and the parameters of the rolling process. The 


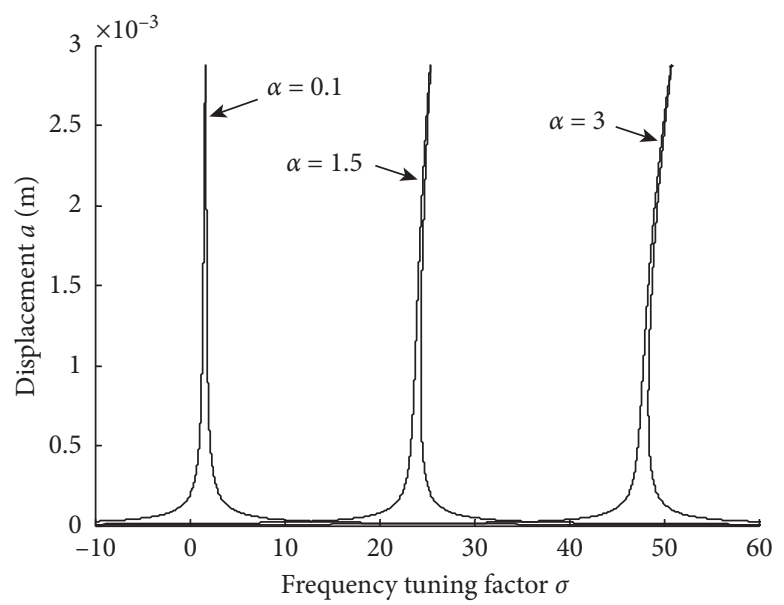

FIGURE 6: Influence of nonlinear spring force coefficient on amplitude frequency characteristic curve.

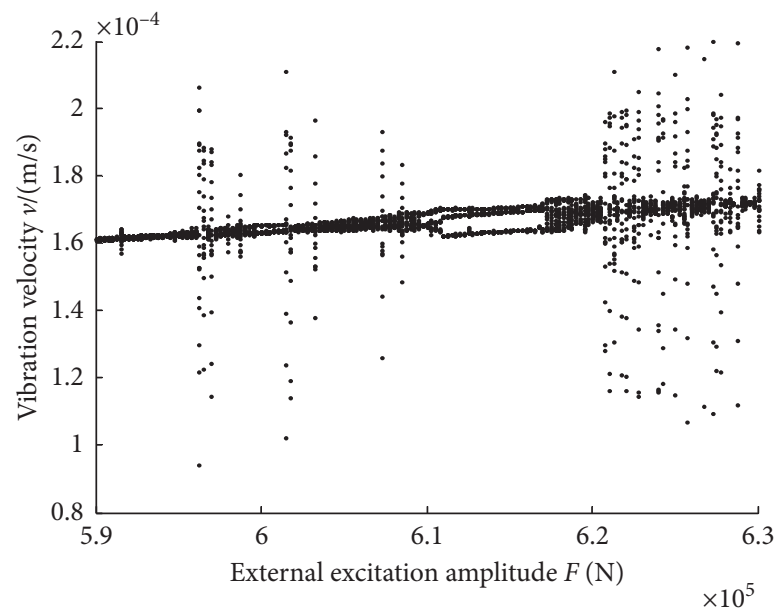

Figure 7: Bifurcation characteristics of rolling mill system.

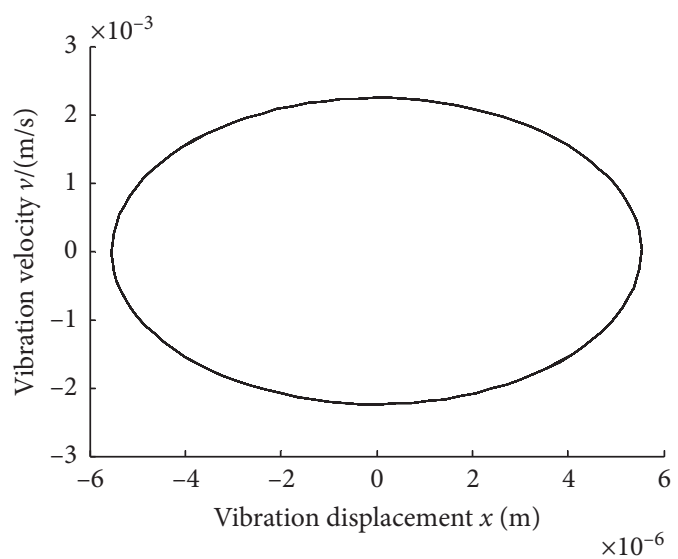

(a)

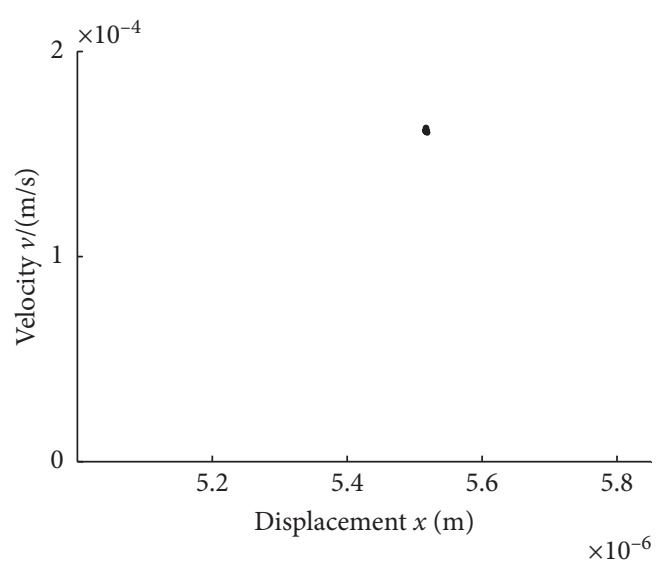

(b)

Figure 8: $F=5.91 \times 10^{5} \mathrm{~N}, 1$ time cycle movement. (a) Phase trajectory diagram. (b) Poincaré section. 


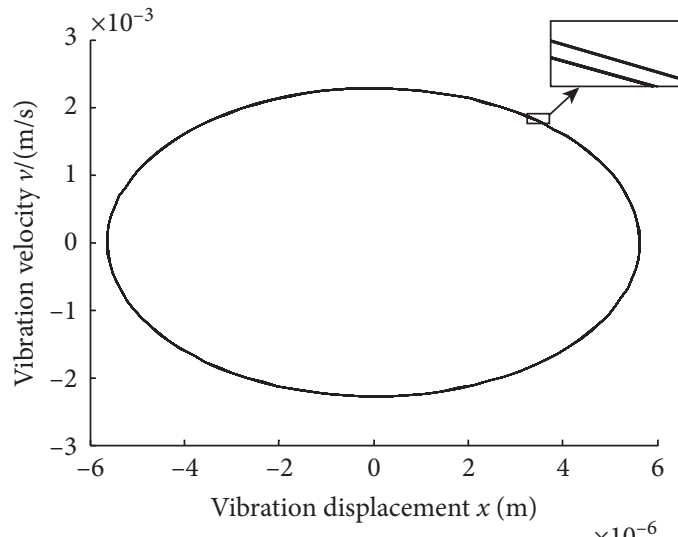

(a)

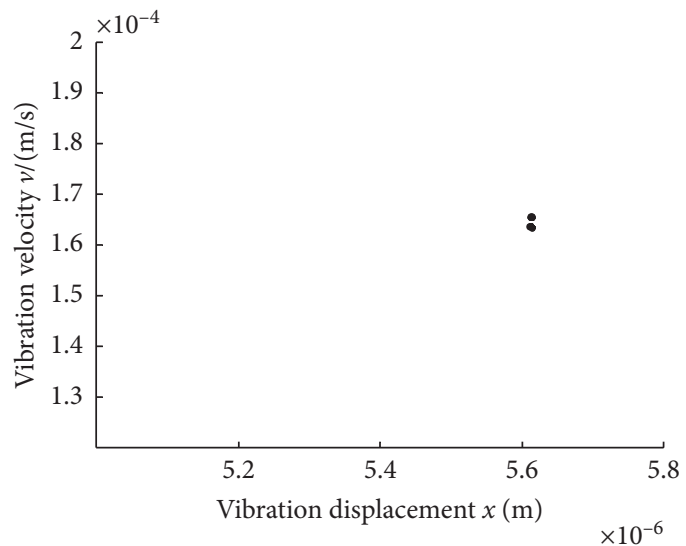

(b)

Figure 9: $F=6.012 \times 10^{5} \mathrm{~N}, 2$-time cycle movement. (a) Phase trajectory diagram. (b) Poincaré section.

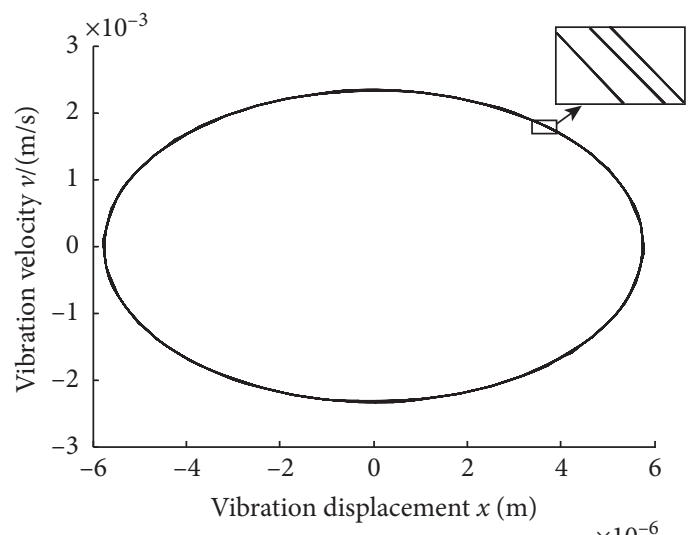

(a)

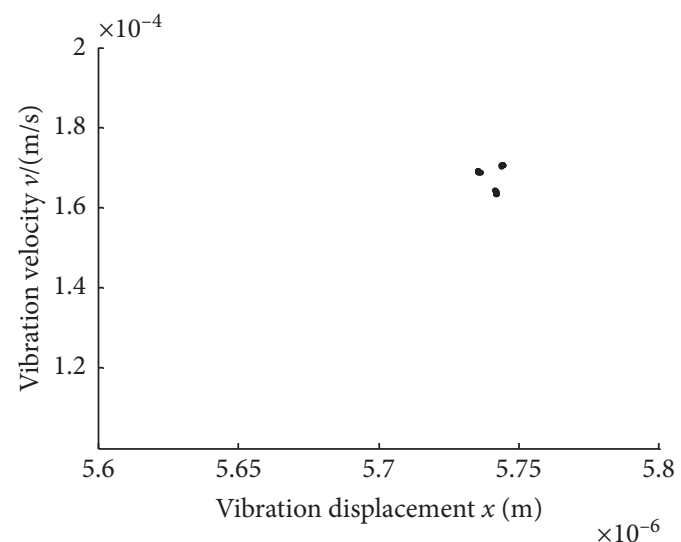

(b)

Figure 10: $F=6.15 \times 10^{5} \mathrm{~N}, 3$-time cycle movement. (a) Phase trajectory diagram. (b) Poincaré section.

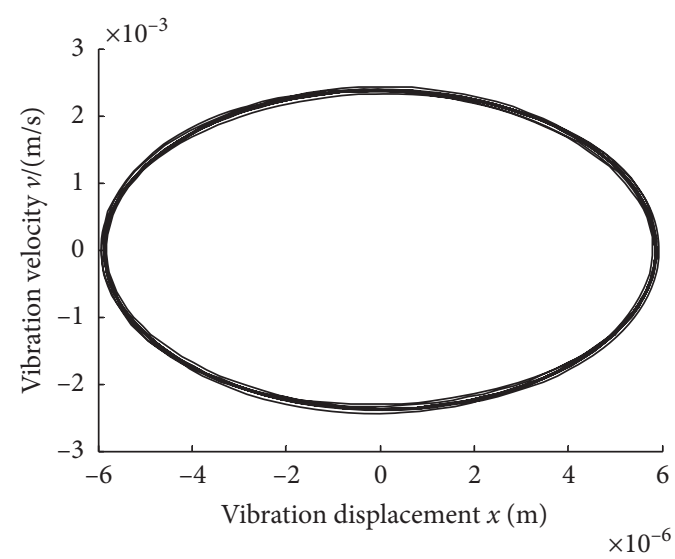

(a)

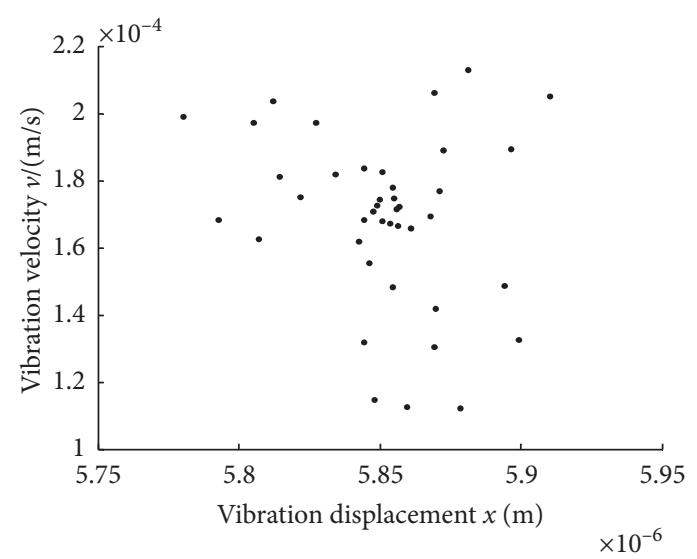

(b)

Figure 11: $F=6.27 \times 10^{5} \mathrm{~N}$, chaotic motion. (a) Phase trajectory diagram. (b) Poincaré section. 
simulation experiments are consistent with the actual working process of the rolling mill, and the simulation results reflect the characteristics of the rolling mill.

As to the limitations of the passive rotational magnetorheological (MR) damper, the need of keeping the electric current continuously in the damper, the strategy of using a discontinuous signal of the current proved to be a good alternative [27]. Through the simulation experiment of actual rolling mill parameters, the data basis for improving the stability of rolling mill vibration is provided. In the actual production process, the vibration of rolling mill can be suppressed by adjusting the external excitation to reduce the influence of vibration on the quality of rolling piece. Due to the special structure of the 1780 mill system, the quality of the rolling mill system, the spring stiffness, and the friction coefficient of the hydraulic cylinder cannot be changed. The actual experiment cannot test the vibration law of the rolling mill by changing these parameters. At present, it is difficult to test in the field, which can only capture the time-domain characteristic and weak spectrum characteristic of the rolling mill vibration. By using the real parameters of the 1780 rolling mill, the characteristics of the nonlinear spring force and the nonlinear friction force on the rolling mill are obtained. The amplitude frequency characteristics and bifurcation characteristics obtained from the simulation experiments can be used to analyze the characteristics of the working process of the rolling mill. The results of simulation experiment are applied to the field experiment, and the structural parameters and process parameters are adjusted according to the simulation results.

\section{Conclusions}

Based the nonlinear spring force and friction force of the hydraulic cylinder, the vibration model of rolling mill system is established. The dynamic equations of the system are constructed, and then the amplitude frequency response equations are obtained. The simulation analysis is carried out from three aspects: the time domain, the amplitude frequency, and the bifurcation characteristic. Through the analysis of numerical simulation, the main conclusions are obtained as follows:

(1) Increasing the nonlinear friction force and spring force lead to the irregular change of the vertical vibration curve of rolling mill system. With the increase of nonlinear force, the rolling mill system shows irregular vibration, which affects the stability of the system.

(2) The initial position of the hydraulic cylinder is changed, which affects the size of the natural frequency of the rolling mill and the range of the stable region of the system; the increase of nonlinear friction force restrains the vibration of rolling mill system; the increase of the nonlinear spring force makes the unstable region of the roller system of rolling mill to become wider, and the stability of the system is affected.
(3) Under different excitation amplitude, the vibration of rolling mill system is alternating between periodic motion, period-doubling motion, and chaotic motion. The dynamic behavior of roll system can be controlled by changing the amplitude of the external excitation.

\section{Data Availability}

The data used to support the findings of this study are available from the corresponding author upon request.

\section{Conflicts of Interest}

The authors declare that they have no conflicts of interest.

\section{Acknowledgments}

This work was supported by the Natural Science Foundation of Hebei Province, China (Grant no. E2015203349).

\section{References}

[1] J. Niziol and A. Swiatoniowski, "Numerical analysis of the vertical vibrations of rolling mills and their negative effect on the sheet quality," Journal of Materials Processing Technology, vol. 162, no. 1, pp. 546-550, 2005.

[2] E. Brusa and L. Lemma, "Numerical and experimental analysis of the dynamic effects in compact cluster mills for cold rolling," Journal of Materials Processing Technology, vol. 209, no. 5, pp. 2436-2445, 2009.

[3] S. Wu, Y. Shao, L. Wang, Y. Yuan, and C. K. Mechefske, "Relationship between chatter marks and rolling force fluctuation for twenty-high roll mill," Engineering Failure Analysis, vol. 55, no. 1, pp. 87-99, 2015.

[4] A. Heidari and M. R. Forouzan, "Optimization of cold rolling process parameters in order to increasing rolling speed limited by chatter vibrations," Journal of Advanced Research, vol. 4, no. 1, pp. 27-34, 2013.

[5] S. K. Yildiz, J. F. Forbes, B. Huang et al., "Dynamic modelling and simulation of a hot strip finishing mill," Applied Mathematical Modelling, vol. 33, no. 7, pp. 3208-3225, 2009.

[6] J. Zhao, X. Wang, Q. Yang, Q. Wang, C. Liu, and G. Song, "High precision shape model and presetting strategy for strip hot rolling," Journal of Materials Processing Technology, vol. 265, no. 3, pp. 99-111, 2019.

[7] Y. Yang and Y. Peng, "Dynamic rolling model based on uniform deformation," Journal of Manufacturing Processes, vol. 58, no. 10, pp. 1334-1347, 2020.

[8] I. Yarita, K. Furukawa, Y. Seino, T. Takimoto, Y. Nakazato, and K. Nakagawa, "An analysis of chattering in cold rolling for ultrathin gauge Steel strip," Transactions of the Iron and Steel Institute of Japan, vol. 18, no. 1, pp. 1-10, 1978.

[9] J.-L. Sun, Y. Peng, and H.-M. Liu, "Non-linear vibration and stability of moving strip with time-dependent tension in rolling process," Journal of Iron and Steel Research International, vol. 17, no. 6, pp. 11-15, 2010.

[10] I. S. Yun, W. R. D. Wilson, and K. F. Ehmann, "Review of chatter studies in cold rolling," International Journal of Machine Tools and Manufacture, vol. 38, no. 12, pp. 14991530, 1998.

[11] X. Yang, Q. Li, C. Tong et al., "Vertical vibration model for unsteady lubrication in rolls-strip interface of cold rolling 
mills," Advances in Mechanical Engineering, vol. 4, no. 12, pp. 1432-1437, 2012.

[12] X. Fan, Y. Zang, and H. Wang, "Research on hot rolling mill horizontal vibration," China Mechanical Engineering, vol. 21, no. 15, pp. 1801-1804, 2010.

[13] A. Bayón, F. Gascón, R. Medina, F. J. Nieves, and F. J. Salazar, "On the flexural vibration of cylinders under axial loads: numerical and experimental study," Journal of Sound and Vibration, vol. 331, no. 10, pp. 2315-2333, 2012.

[14] L. Wang and B. Wu, R. Du et al., "Nonlinear dynamic characteristics of moving hydraulic cylinder," Chinese Journal of Mechanical Engineering, vol. 43, no. 12, pp. 12-19, 2007.

[15] Z. Gao, L. L. Bai, and Q. LI, "Research on critical rolling speed of self-excited vibration in the tandem rolling process of thin strip," Journal of Mechanical Engineering, vol. 53, no. 12, pp. 118-132, 2017.

[16] B. Liu, G. X. Pan, P. LI et al., "Vibration analysis of roll system with nonlinear stiffness of hydraulic cylinder," Iron and Steel, vol. 52, no. 3, pp. 93-99, 2017.

[17] W. Zhang and X. Yang, "Transverse nonlinear vibrations of a circular spinning disk with a varying rotating speed," Science China Physics, Mechanics and Astronomy, vol. 53, no. 8, pp. 1536-1553, 2010.

[18] W. Zhang, R. Zhou, and W. Zu Jean, "Nonlinear vibrations of a shell-shaped workpiece during high-speed milling process," Nonlinear Dynamics, vol. 40, no. 1, pp. 767-787, 2013.

[19] R. Zhou, W. Zhang, and J. W. Zu, "Analysis on nonlinear dynamics of a thin-plate workpiece in milling process with cutting force nonlinearities," Journal of Mechanical Science and Technology, vol. 28, no. 7, pp. 2511-2526, 2014.

[20] P. Andrea, P. Luca, V. Francesco et al., "Design and testing of an innovative electro-hydraulic actuator for a semi-active differential," in Proceedings of the Institution of Mechanical Engineers, Part D: Journal of Automobile Engineering, vol. 232, no. 11, pp. 1438-1453, 2017.

[21] M. C. Valigi, M. Malvezzi, and S. Logozzo, "A numerical procedure based on Orowan's theory for predicting the behavior of the cold rolling mill process in full film lubrication," Lubricants, vol. 8, pp. 1-13, 2020.

[22] P.-H. Hu and K. F. Ehmann, "A dynamic model of the rolling process. Part I: homogeneous model," International Journal of Machine Tools and Manufacture, vol. 40, no. 1, pp. 1-19, 2000.

[23] I.-S. Yun, W. R. D. Wilson, and K. F. Ehmann, "Chatter in the strip rolling process, Part 1: dynamic model of rolling," Journal of Manufacturing Science and Engineering, vol. 120, no. 2, pp. 330-336, 1998.

[24] J.-Y. Oh, G.-H. Jung, G.-H. Lee, Y.-J. Park, and C.-S. Song, "Modeling and characteristics analysis of single-rod hydraulic system using electro-hydrostatic actuator," International Journal of Precision Engineering and Manufacturing, vol. 13, no. 8, pp. 1445-1451, 2012.

[25] Y. Zhu, W. Jiang, S. Liu et al., "Research on influences of nonlinear hydraulic spring force on nonlinear dynamic behaviors of electro-hydraulic servo system," China Mechanical Engineering, vol. 26, no. 8, pp. 1085-1091, 2015.

[26] H. Shi, Vibration system, Huazhong University of Science \& Technology Press, Wuhan, China, 2004.

[27] A. Tusset, F. Janzen, V. Piccirillo, R. Rocha, J. Balthazar, and G. Litak, "On nonlinear dynamics of a parametrically excited pendulum using both active control and passive rotational (MR) damper," Journal of Vibration and Control, vol. 24, no. 9, pp. 1587-1599, 2018. 